\title{
Molecular insights into the regulation of rice kernel elongation
}

\begin{abstract}
A large number of rice agronomic traits are complex, multi factorial and polygenic. As the mechanisms and genes determining grain size and yield are largely unknown, the identification of regulatory genes related to grain development remains a preeminent approach in rice genetic studies and breeding programs. Genes regulating cell proliferation and expansion in spikelet hulls and participating in endosperm development are the main controllers of rice kernel elongation and grain size. We review here and discuss recent findings on genes controlling rice grain size and the mechanisms, epialleles, epigenomic variation, and assessment of controlling genes using genome-editing tools relating to kernel elongation.
\end{abstract}

Keyword: Kernel elongation; Cell proliferation and expansion; Rice grain size; Epialleles and epigenomic; Genome editing 